\title{
Neurosyphilis Presenting with Optic Neuritis
}

\author{
R. Arvinth, Z. Mimiwati, and S. C. Reddy
}

\section{ABSTRACT}

The incidence of syphilis has greatly reduced in the severity of affected individuals due to the early treatment with antibiotics. However, due to the increase in the prevalence of human immunodeficiency virus (HIV) infection, it has also caused a concurrent rise in the number of neurosyphilis patients. Most common ocular manifestations include uveitis, interstitial keratitis, and vasculitis. A healthy 28 -years old man presented with a progressive blurring of vision in the right eye for one month. It was his second episode. The first episode occurred 4 months back which resolved spontaneously without treatment. Vision in the right eye was $6 / 18$. The pupil was dilated and the relative afferent pupillary defect was positive. Fundus examination showed a hyperemic swollen right optic disc with blurred margins and no macular fan or star. Vision, anterior segment, and fundus were normal in the left eye. After the investigations, he was diagnosed as a case of neurosyphilis with optic neuritis. He was treated with intravenous penicillin for two weeks followed by oral penicillin for three weeks. Vision in the right eye improved to $6 / 6$; the optic disc swelling resolved with clear margins. He maintained good vision during the follow-up of six months. Early referral to an ophthalmologist upon suspicion of syphilis, detailed evaluation, and immediate treatment is mandatory to prevent permanent vision loss in these patients.

Keywords: Optic neuritis, neurosyphilis, penicillin.
Published Online: October 12, 2021

ISSN: $2736-5476$

DOI: $10.24018 /$ ejclinicmed.2021.2.5.126

R. Arvinth

Department of Ophthalmology, Faculty of Medicine, University of Malaya, Kuala Lumpur, Malaysia.

Department of Ophthalmology, Hospital Ampang, Selangor, Malaysia.

Z. Mimiwati

Department of Ophthalmology, Faculty of Medicine, University of Malaya, Kuala Lumpur, Malaysia.

S. C. Reddy*

Department of Ophthalmology, Faculty

of Medicine and Defence Health,

National Defence University of Malaysia,

Kuala Lumpur, Malaysia.

(e-mail: profscreddy@gmail.com)

*Corresponding Author

\section{INTRODUCTION}

Syphilis is a chronic, systemic infection transmitted by spirochaete Treponema Pallidum. Humans are the only known natural hosts of this organism. Systemically, it can cause chronic inflammation of the central nervous system resulting in headache, stiffness of the neck, nausea, vomiting, dementia [1], [2], and psychiatric symptoms such as depression, mania, psychosis, and personality changes [3]. It also causes ocular manifestations such as interstitial keratitis, anterior, intermediate, and posterior uveitis, chorioretinitis, retinitis, vasculitis, cranial nerve, and optic neuropathies [4]. Neurosyphilis is diagnosed with a cerebrospinal fluid (CSF) white blood cell (WBC) count of 20 cells $/ \mu \mathrm{L}$ or greater, or a reactive CSF Venereal Disease Research Laboratory (VDRL) test result. It can occur at any stage of syphilis. Presumptive neurosyphilis is suspected at any stage of syphilis in the presence of a non-reactive CSF VDRL, when we find an increase in CSF protein or WBC count with possible clinical signs and symptoms suggesting neurosyphilis [1]. Due to the wide range of manifestations caused, syphilis is commonly known as the 'great imitator'. Diagnosis is by strong clinical suspicion supported by treponemal and non-treponemal serologic tests. All patients with diagnosed syphilis should be tested for HIV co-infection due to the higher incidence of disease progression in the immunocompromised people. However, the incidence of neurosyphilis has drastically reduced currently due to early intervention and treatment with appropriate antibiotics [3]-[5].

We report an atypical presentation of optic neuritis in a young Malay gentleman caused by neurosyphilis which presented with visual deterioration. Previous case reports published in Malaysia had similar presentations; however, they were associated with HIV positive [6], [7], in contrast to our patient who was HIV negative and otherwise well. Previous case reports of neurosyphilis involving the eye in Malaysia had other findings such as panuveitis, anterior or posterior uveitis [7], [8], while our patient had signs of optic neuritis only with no other ocular features. This made the diagnosis challenging for us. To our best knowledge, this is the first time that syphilitic optic neuritis as a sole ocular presentation in neurosyphilis has been reported in Malaysia. The patient regained full vision after the treatment with intravenous and oral penicillin.

\section{CASE REPORT}

A healthy 28-years old man presented with progressive right eye blurring of vision for one month. It was his second episode. The first episode occurred 4 months back which resolved spontaneously without treatment. There was no diplopia, fever, trauma, intravenous drug abuse, or drug history intake. The patient had initially denied being involved in any sexual activities.

On ocular examination, vision in the right eye was $6 / 18$, not improving with pinhole, and in the left eye was $6 / 6$. The 
right pupil was dilated and sluggishly reacting to light. Relative afferent pupillary defect (RAPD) was positive grade 3 in the right eye, with no Argyll-Robertson pupil. Light brightness and red desaturations were $30 \%$ and $20 \%$ respectively in the right eye. Ishihara color vision charts testing revealed red-green deficiency. Otherwise, extraocular muscle movements, anterior segment, and intraocular pressure were normal. Fundus examination showed hyperemic, swollen (2 diopters) right optic disc with blurred margins (Fig. 1). No features of neuroretinitis (macular fan or star) were seen in the macula. Left eye fundus was normal. Humphry visual field testing (30 degrees) showed an enlarged blind spot with central scotoma in the right eye. The left eye visual field was normal.

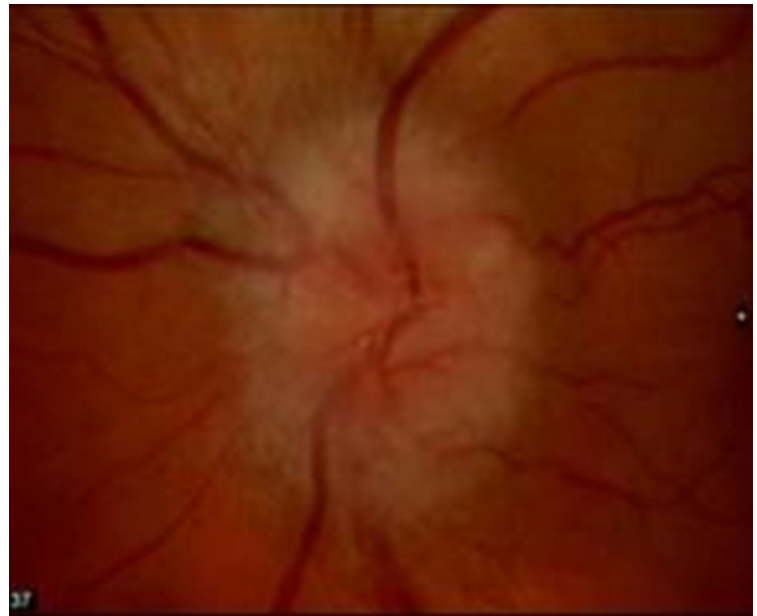

Fig. 1. Fundus photo showing swollen right optic disc with blurred margins at presentation.

There were no palpable lymph nodes or ulcers around the genitalia. The patient was admitted to the eye ward for investigations and treatment. Full blood count and renal profile were within normal limits. A rapid plasma reagin (RPR) test was positive for syphilis with a high titer of 1:256. Enzyme linked immunosorbent assay (ELISA) revealed elevated total immunoglobulin $\mathrm{M}$ antibodies against Treponema pallidum. Hepatitis B, C; and HIV were negative. There were no infective foci detected in the lungs on the chest X-ray. Magnetic Resonance Imaging (MRI) of the brain and orbit revealed no evidence of demyelinating disease. Mantoux test was non-reactive.

The patient was notified of the findings and upon counseling further, the patient confessed to having multiple sexual partners of the same sex for the past 1 year. He experienced a few genital ulcers 2 months ago which had resolved spontaneously without treatment.

Lumbar puncture was performed, and the CSF sample was sent for biochemical analysis. Although the CSF for VDRL testing was negative for Treponema pallidum, we detected leukocytosis of 15 cells $/ \mu \mathrm{L}$ with mildly elevated CSF VDRL protein level. However, glucose levels were normal.

The patient was treated as having optic neuritis secondary to neurosyphilis. He was started on benzyl penicillin 3M units 4 hourly intravenously for 2 weeks. The patient experienced a gradual improvement of vision along the course of treatment. Upon completing antibiotic treatment, the patient's vision improved to 6/6. RAPD was positive grade 1 .
Fundoscopy showed reduced hyperemia of the right optic disc. The patient was discharged from the hospital on oral Penicillin- V $500 \mathrm{mg}$ two times daily for 3 weeks. In the follow-up examination after completion of the treatment, vision in the right eye maintained 6/6, with minimal detectable RAPD. Red desaturation and light brightness had improved to $90 \%$. Fundus examination of the right eye revealed complete resolution of the hyperemia and blurred margins of the optic disc (Fig. 2). Since there was improvement and maintenance of vision with penicillin therapy, the oral corticosteroids were not started in this patient. He was followed up once in two months for 6 months. In the last follow-up examination, vision in the right eye remained 6/6; anterior segment and fundus were normal, and the patient was asymptomatic.

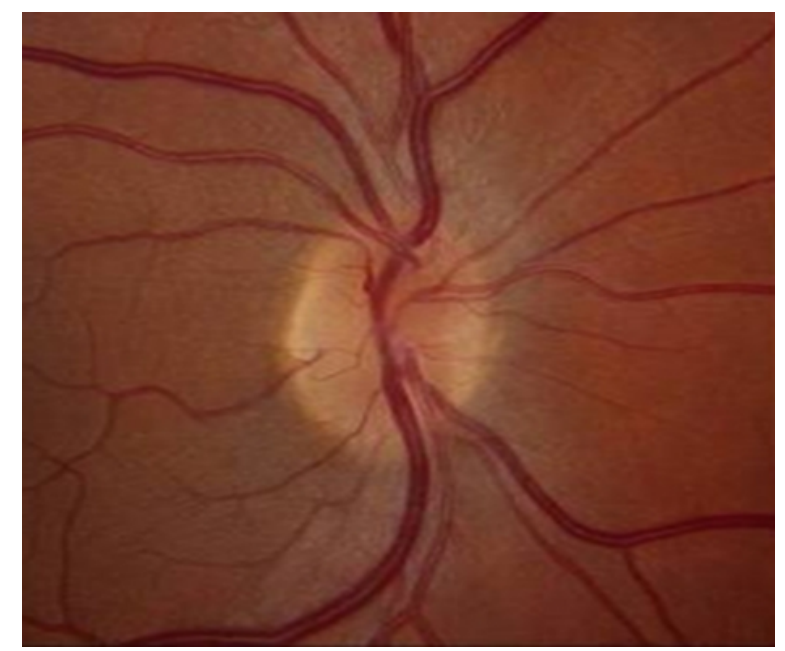

Fig. 2. Fundus photo showing normal right optic disc with clear margins after completion of penicillin treatment.

\section{DISCUSSION}

Treponema pallidum, the causative organism in syphilis, penetrates the skin through abrasion and spreads through blood and lymph. Primary infection occurs following an incubation period of 3 weeks. A painless ulcer (primary chancre) develops at the site of infection which heals spontaneously [1], [2]. Secondary infections occur 4-8 weeks after primary infection. However, in certain cases, Treponema pallidum can remain dormant in the body before causing infection [9].

Syphilis involves the nervous system [1], [2] and presents with ophthalmological signs in approximately $18 \%$ of patients [10]. However, the incidence of optic neuritis due to syphilis is relatively uncommon. Our patient was treated as presumed neurosyphilis based on the CSF findings despite not presenting with neurological features of neurosyphilis. There are no characteristic features of syphilitic optic neuritis to differentiate it from non-syphilitic causes. However, it is important to perform an MRI to rule out multiple sclerosis or neuromyelitis optica, which was performed in our patient. Both these conditions can co-exist, necessitating a different treatment modality [11]. Dhungana et al. [12] reported that syphilis can progress to syphilitic optic neuropathy if left untreated as evidenced by poor vision and RAPD in our patient. A study by $\mathrm{Gu}$ et al. [13] showed that delayed 
treatment for syphilitic optic neuropathy can lead to significant permanent visual loss. Hence, all patients with optic neuritis should be tested for syphilis by definitive investigations.

However, in neurosyphilis particularly involving the eye, CSF cellularity may be normal or elevated and the CSF VDRL may be negative [14]. In our patient, the diagnosis of optic neuritis due to syphilis was difficult to make initially, due to inaccurate history and the absence of classical chancre on the genitalia or skin ulcers seen during the initial examination. As such, treating practitioners, especially at the first contact with the patient, should take a detailed history and perform a thorough clinical examination [15]. The CSF glucose level, which we would have expected to be decreased [16], was possibly normal indicating an extremely early stage of the disease.

However, false positives conditions such as pregnancy and false negative conditions such as concomitant HIV infection can influence the result. Hence, if clinical suspicion of neurosyphilis is high despite a negative CSF, it is recommended that repeat testing be done to reduce the likelihood of obtaining an inaccurate result [17]. Although highly specific, CSF VDRL is not sensitive and can be negative in $50 \%$ of patients with active neurosyphilis [1], [17]. Administration of benzathine penicillin $G$ is beneficial in patients with ocular syphilis, even when they are coinfected with HIV [18]. Ocular syphilis is considered a neurosyphilis variant, meaning the primary treatment for ocular syphilis is administering IV benzyl penicillin for 14 days [19]. This practice adheres to the Malaysian clinical practice guidelines and WHO guidelines for the treatment of neurosyphilis [20], [21].

\section{CONCLUSION}

The incidence of syphilis causing complications has reduced tremendously in view of the availability of better medical care facilities. However, delayed treatment can cause complications. The difficulty in the initial diagnosis and subsequently delayed treatment could probably be due to the inaccurate history given by the patient. Although optic nerve was involved, the patient responded well to antibiotic treatment and regained full vision. The patient was advised to get his fellow sexual partners to perform a syphilis screening as it can spread sexually.

\section{REFERENCES}

[1] R. P. Knudsen and N. N. Singh, "Neurosyphilis Overview of Syphilis of the CNS," http://emedicine.medscape.com/article/1169231overview, Updated Jul 17, 2018. (Accessed on Aug. 31, 2021)

[2] J. R. Berger and D.Dean, "Neurosyphilis," Handb Clin Neurol 121, 1461-72, 2014

[3] E. Akinci, F. Oncu and B. Topcular, "Neurosyphilis in psychiatric settings: Three Case Reports," Turk Psikiyatri Derg 28(1): 61-66, 2017.

[4] S. Kiss, F. M. Damico and L. H. Young, "Ocular manifestations and treatment of syphilis," Semin Ophthalmol 20(3): 161-167, 2005

[5] T. Toptan, B. Ozdilex, G. Kenangil, M. Ulker and F. Mayda Domac, "Neurosyphilis: a case report," North Clin Istanb 2(1): 66-68, 2015.

[6] A. Hanifatiyah, A. Mohd Fairuz, A. T. Nor Azita and R. O. Raja Nor Farahiyah, "Altered Vision in MSM Patient, Diagnosis of Ocular Syphilis in Primary Care: A Case Report," Mal J Med Health Sci 15(1): $85-88,2019$.
[7] M. Mustapha, Z. Abdollah, A. Ahem, H. Mohd Isa, M. L. C. Bastion and N. M. Din, "Ocular syphilis: resurgence of an old disease in modern Malaysian society," Int J Ophthalmol 11(9):1573-1576, 2018.

[8] A. K. Ghanimi Zamli, N. S. Irma Ngah, T. Chew-Ean, J. Muhammad, W. H. Wan Hittam, A. Hussein and E. Zunaina, "Clinical Profile and Visual Outcomes of Ocular Syphilis: A Five-year Review in Hospital Universiti Sains, Malaysia," Cureus 11(2): e4015, 2019.

[9] N. Farai and T.Craig, "Syphilis: presentations in general medicine," Clin Med (London) 16(2): 184-188, 2016.

[10] G. T. Smith, D. Goldmeier and C.Migdal, "Neurosyphilis with optic neuritis: An update," Postgrad Med J 82(963): 36-39, 2006.

[11] F. Shan, Y. Long, Y. Fan, M. Chen, Y. Zheng and C. Gao, "Neuromyelitis optica spectrum disorder and neurosyphilis coexist in a chinese woman," Journal of Molecular Biomarkers \& Diagnosis 5(6), 198, 2014.

[12] S. Dhungana, B. Shararack, I. Pepper and J. Bowen, "Syphilitic Optic Neuropathy: A Diagnosis Not to Be Missed," The Internet Journal of Neurology 9(2): 25-30, 2007.

[13] X. Gu, Y. Gao, Y. Yan, M. Marks, L. Zhu, H. Lu and P. Zhou, "The importance of proper and prompt treatment of ocular syphilis: a lesson from permanent vision loss in 52 eyes," Journal of the European Academy of Dermatology and Venereology 34(7): 1569-1578, 2020.

[14] L. Porto, J. Capelo and A. Carragoso, "Unilateral swollen optic disc: do not forget neurosyphilis," BMJ Case Rep 3:31-35, 2013.

[15] G. Gaurav, J. O. Jason, Y. C. Marcus and L. L. Lyndell, "Keeping an eye on syphilis," 46(6): 401- 404, 2017.

[16] M. Pastuszczak, A. Wojas-Pelc and A. Jaworek, "Association of CSF glucose concentration with neurosyphilis diagnosis," Open Medicine 8(1): 48-51, 2013.

[17] L. Yong, K. Wujian, Y. LiGang and W. Zhenyu, "Clinical prediction and diagnosis of neurosyphilis in HIV-negative patients: a case-control study," BMC Infectious Diseases 19(1): 1-7, 2019.

[18] V. C. Fiona and F.Martin, "Management of syphilis in HIV-positive individuals," Sex Health 12(2):135-40, 2015.

[19] S. L. Woolston, S. Dhanireddy and J. Marrazzo, "Ocular syphilis: a clinical review," Curr Infect Dis Rep 18:36, 2016.

[20] Health Management and Disease Control, Ministry of Health: Malaysian Guidelines in the Treatment of Sexually Transmitted Infection, $4^{\text {th }}$ ed, Malaysia; 2015.

[21] World Health Organization. Guidelines for the management of sexually transmitted Infections 2003. https://apps.who.int/iris/handle/10665/ 42782. (Accessed on Sept.10, 2021). 